\title{
Nonlinear Distortion Cancellation in Spatial Division Multiplexing System Based on RZ-Coded PCTWs Technique
}

\author{
Shahad S. Husain and Jassim K. Hmood \\ Laser and Optoelectronics Eng. Dept., University of Technology, Baghdad, Iraq. \\ shahadsabah10@gmail.com140011@uotechnology.edu.iq
}

\begin{abstract}
SDM) system has been considered as promising systems due to its ability to transport a higher bit rate for longer transmission distance. However, nonlinear phase noise (NPN (degrades SDM system performance. In this paper, we propose return-to-zero (RZ)-coded phase-conjugated twin waves (PCTWs) to improve the effectiveness of nonlinear distortion cancellation in the SDM system. In this approach, the PCTWs are modulated by m-array quadrature amplitude modulation (mQAM) then RZ encoded. After that, RZ-mQAM PCTWs are co-propagated over two fibres links. The received signals are superimposed to suppress the NPN. An analytical model that characterizes the performance of spatial-multiplexed (SM) RZ-coded PCTWs scheme is developed. Moreover, we numerically investigate the system performance with 4QAM format at 20Gsymbol/s rate. The results display that the performance is substantially better for the proposed scheme. Transmission reaches of both 4QAM PCTWs and RZ-4QAM PCTWs schemes are extended by $77.8 \%$ and $100 \%$, respectively, in contrast with the 4QAM scheme.
\end{abstract}

Index Terms-Fibre nonlinearity, Nonlinear distortion, Phase-conjugated twin waves technique, Spatial division multiplexing system.

\section{INTRODUCTION}

Recently, SDM systems have extensively studied due to their ability to transport high-speed information [1]-[4]. To enhance the spectral efficiency of SDM systems, multi-level modulation such as mQAM and m-array phase shift keying are utilized [5], [6]. However, the performance of coherent SDM systems is degraded by nonlinear phase noise (NPN). Self-phase modulation (SPM) is created by Kerr effects once the optical wave transport over fibre [7], [8]. Stochastic impairments can be induced when nonlinear impairments interact with the amplifier's noise. A random NPN is evoked when amplified spontaneous emission (ASE) noise is mixed with the SPM phenomenon [9]. Various parameters significantly rise NPN, for example, higher signal power, broad communication reach, and amplifiers number [8], [10].

Different transmission techniques have been effectively developed to enable coherent communication systems to transmit information for longer reach with a good signal-to-noise ratio (SNR) [11], [12], [8], [13]. The optical parametric oscillation has been utilized to cancelling the NPN in the optical phase conjugation (OPC) method [14], [15]. Regardless, the efficiency of nonlinear suppressing of the OPC technique is restricted by symmetry conditions that can limit network flexibility. flexibility. Other techniques based on coding or interleaving optical signals have been proposed for mitigating fiber nonlinearity. RZ code has been employed in single and multichannel optical telecommunication systems [16], [17]. Besides, coding high-order modulation formats by RZ 
code was studied to increase nonlinearity tolerance in all-optical orthogonal frequency division multiplexing system [18]-[20]. Interleaving technique was used to lower fiber nonlinearity based on lessening signals interference. For example, polarization interleaving method was carried out to decrease interference among optical signals [17], [21]. Nevertheless, polarization mode dispersion (PMD) extremely influenced the efficiency of this technique.

Over the last few years, the PCTWs method has been implemented in two orthogonal dimensions to cancel NPN[11]. Symmetrical dispersion map is the main condition to get the full effectiveness of the PCTWs technique [22], [23]. Really, various types of orthogonal domains have been exploited for implementing PCTWs method. Firstly, the PCTWs method has been carried by orthogonal polarization states [24], [11]. But, PMD decrease the PCTWs influence. Besides, PCTWs have been transmitted over neighboring time slots that effectively reduces nonlinear impairments [25]. The PCTWs method was also investigated in the frequency dimension by guiding the waves by adjacent channels [22], [26]. Additionally, the PCTWs method has been investigated in the spatial division multiplexing system [26][28]. Still, the PCTWs technique cannot cancel the random part of nonlinear distortion, which is produced when the nonlinear effects interact with the amplifier's noise [29].

In this paper, we proposed a new approach for enhancing the effectiveness of PCTWs method in mQAM SDM system by shaping the envelope of PCTWs using RZ coding. In this method, the twin waves are modulated by mQAM and coded using RZ encoder. Consequentially, they co-propagated over two similar optical links. At the reception, received signals are synchronously superimposed to improve the performance of the system. Further, nonlinear distortion cancellation in RZ-mQAM system is analytical modeled and numerically investigated. The results denote that nonlinearity tolerance of the proposed scheme is greatly enhanced in comparison with the 4QAM system. In addition, for the proposed scheme, the communication reach is lengthed by $100 \%$, and SNR is improved by $\sim 6.5 \mathrm{~dB}$ in contrast to the 4QAM system.

\section{THE SYSTEM MODELLING}

To describe the effect of shaping the envelope of twin waves on NPN in the SM PCTWs scheme, a model is analytically derived. Figure 1 illustrates the simple block diagram that characterizes the proposed system with PCTWs method. The optical wave and its phase-conjugated replica are first modulated by the same laser beam and then sent over parallel optical links. dispersion compensation fibers(DCFs), Single-mode fiber (SMF), and Erbium-doped fiber amplifier(EDFA) are components of each span. To explore the capability of the proposed scheme to cancel NPN, conventional mQAM systems that included the PCTWs technique are analytically compared. At the transmitter side, the field of the RZ-coded PCTWs is expressed by [28]:

$$
\begin{aligned}
& E(0, t)=\sqrt{\frac{P_{o}}{2}} A(0, t) S(t) \exp \left(\mathrm{j} 2 \pi f_{o} t\right), \\
& E^{*}(0, t)=\sqrt{\frac{P_{o}}{2}} A^{*}(0, t) S(t) \exp \left(\mathrm{j} 2 \pi f_{o} t\right),
\end{aligned}
$$

where represents a power of laser source, indicates the symbol period, symbolizes optical carrier frequency, and are the complex values corresponding to constellation of mQAM wave and its phase-conjugated, respectively. Here, represents the envelope shape of mQAM symbols. Commonly, for mQAM signal, the over symbol interval. To reduce the NPN, the envelope of the transmitted signal can be reshaped by RZ code for minimizing its intensity and interference time with nonlinear effects. Practically, at ultra-high speed transmission 
system, RZ encoder involves one MZM driven by radio frequency oscillator. Thus, the envelope of RZ-mQAM symbol can be described by:

$$
S(t)=\left\{\begin{array}{lc}
\frac{1}{2}\left[1+\sin \left(2 \pi f_{s} t\right)\right] & 0 \leq t \leq T_{s} \\
0 & \text { otherwise }
\end{array}\right.
$$

where $f_{s}=1 / T_{s}$ is a symbol rate. Hence, RZ-mQAM wave is described as:

$$
E_{R Z-m Q A M}(0, t)=\frac{1}{2} \sqrt{\frac{P_{o}}{2}} A(0, t)\left[1+\sin \left(2 \pi f_{s} t\right)\right] \exp \left(\mathrm{j} 2 \pi f_{o} t\right) .
$$

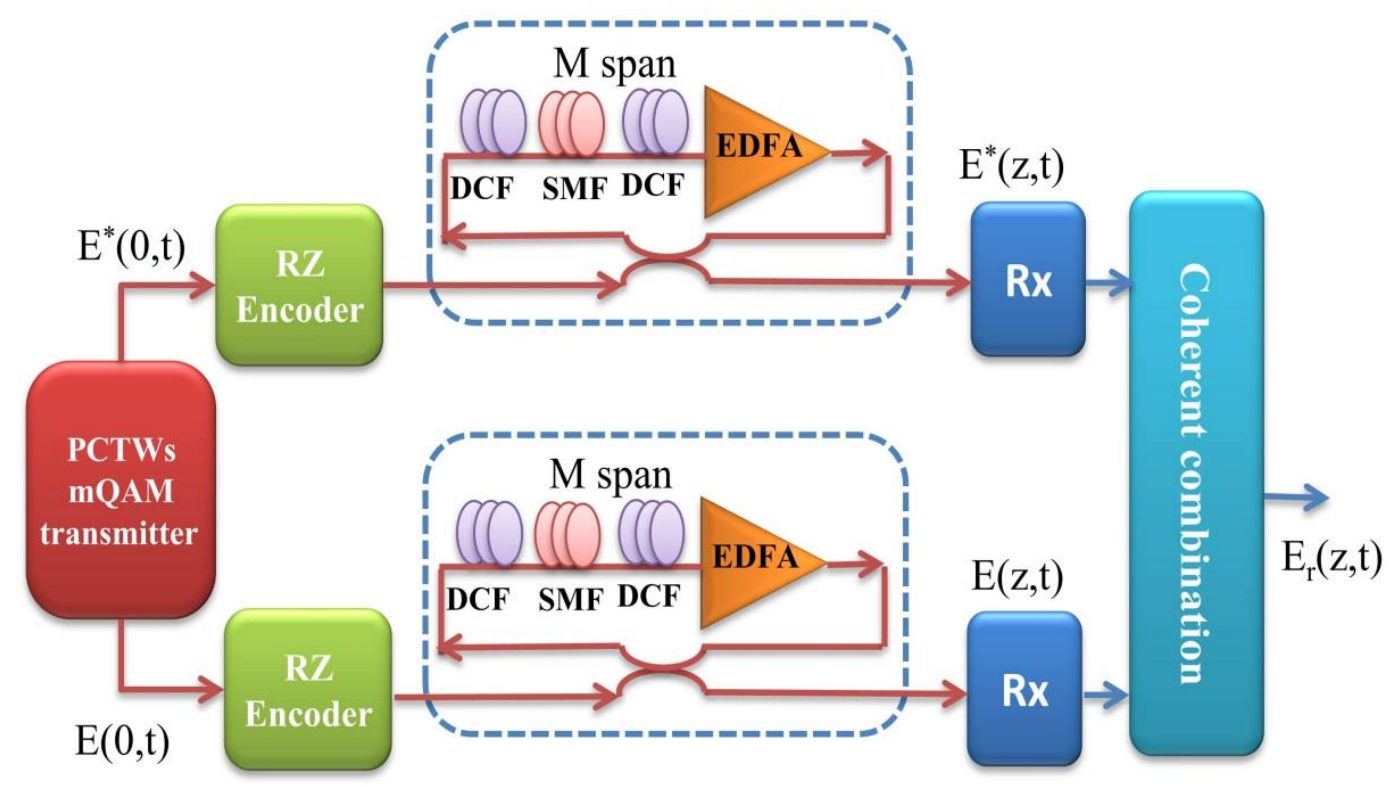

FIG. 1. BLOCK DIAGRAM OF SM RZ-MQAM SCHEME WITH PCTWS METHOD.

In the SM PCTWs scheme, the fiber nonlinearity is mainly formed by SPM where the optical field experiences a nonlinear phase shift that results from its own intensity. For $M$ spans, SPM phase noise and its interaction with amplifier noise can be characterized by[8]:

$$
\theta_{\mathrm{SPM}}^{+A S E}=\gamma L_{e f f}(L) \sum_{m=1}^{M}\left|\mathrm{E}(0, t)+\sum_{v=1}^{m} n(t)_{v}(t)\right|^{2} .
$$

The NPN that added to the transmitted signal at Mth span is written by [26]:

$$
\theta_{\mathrm{SPM}}^{+A S E}=\theta_{S P M}+\theta_{S P M}^{A S E}+\theta^{A S E}
$$

where $\theta_{S P M}$ represents SPM noise, $\theta_{S P M}^{A S E}$ characterizes the random NPN, which is induced by interacting ASE noise with SPM effect and $\theta^{A S E}$ describes the ASE phase noise. By doing some algebra, NPN that produced by SPM only is defined as [26]:

$$
\theta_{S P M}=\left.\gamma L_{e f f} \sum_{m=1}^{M} \mathrm{E}(0, t)\right|^{2}
$$


where $\gamma$ represents nonlinear coefficient, $L_{\text {eff }}=[1-\exp (-\alpha L)] / \alpha$ denotes effective fiber length, $\mathrm{L}$ and $\alpha$ are fiber length and attenuation coefficient. The power content of RZmQAM and mQAM signals are [19]:

$$
\begin{aligned}
& \left|\mathrm{E}_{\text {RZ-mQAM }}(0, \mathrm{t})\right|^{2}=\frac{3 \mathrm{P}_{\mathrm{o}}}{16}|\mathrm{~A}(0, \mathrm{t})|^{2}, \\
& \left|\mathrm{E}_{\text {mQAM }}(0, \mathrm{t})\right|^{2}=\frac{\mathrm{P}_{\mathrm{o}}}{2}|\mathrm{~A}(0, \mathrm{t})|^{2},
\end{aligned}
$$

By substituting Eq. (7) in Eq. (6), yields;

$$
\begin{aligned}
& \theta_{\text {SPM_RZ-mQAM }}=\frac{3 \mathrm{M} \gamma \mathrm{P}_{\mathrm{o}} \mathrm{L}_{\text {eff }}}{16}|\mathrm{~A}(0, \mathrm{t})|^{2}, \\
& \theta_{\text {SPM_mQAM }}=\frac{M \gamma \mathrm{P}_{\mathrm{o}} \mathrm{L}_{\text {eff }}}{2}|\mathrm{~A}(0, \mathrm{t})|^{2},
\end{aligned}
$$

The random NPN that produced inside optical fiber for RZ-mQAM and mQAM waves are characterized by:

$$
\begin{aligned}
& \theta_{\mathrm{SPM} \_ \text {RZ-mQAM }}^{\mathrm{ASE}}=\gamma \mathrm{L}_{\text {eff }} \sqrt{\frac{\mathrm{P}_{\mathrm{o}}}{2}}\left[1+\sin \left(\omega_{\mathrm{s}} \mathrm{t}\right)\right] \sum_{\mathrm{m}=1}^{\mathrm{M}} \Re\left\{\mathrm{A}(0, \mathrm{t}) \sum_{\mathrm{v}=1}^{\mathrm{m}} \mathrm{n}_{\mathrm{v}}(\mathrm{t})\right\}, \\
& \theta_{\mathrm{SPM} \_ \text {mQAM }}^{\text {ASE }}=\sqrt{2 \mathrm{P}_{\mathrm{o}}} \gamma \mathrm{L}_{\text {eff }} \sum_{\mathrm{m}=1}^{\mathrm{M}} \mathfrak{R}\left\{\mathrm{A}(0, \mathrm{t}) \sum_{\mathrm{v}=1}^{\mathrm{m}} \mathrm{n}_{\mathrm{v}}(\mathrm{t})\right\},
\end{aligned}
$$

respectively; where $\mathfrak{R}\{\mathrm{y}\}$ represents real part of number (y). Random NPN variances for both systems are characterized by

$$
\begin{aligned}
& \sigma_{\theta_{\text {SPM }}^{\text {ASER }- \text { mQAM }}}^{2}=\frac{3 \mathrm{M}(\mathrm{M}+1)}{8} \gamma^{2} \mathrm{~L}_{\text {eff }}{ }^{2} \mathrm{P}_{\mathrm{o}} \sigma_{\mathrm{ASE}}^{2}|\mathrm{~A}(0, \mathrm{t})|^{2} \text {, }
\end{aligned}
$$

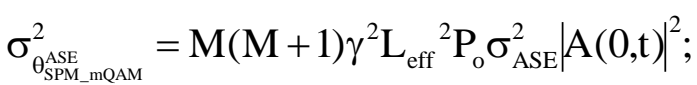

respectively. Variance of amplifiers noises that add to RZ-mQAM and mQAM waves is defined as:

$$
\begin{aligned}
& \sigma_{\theta_{m Q A M}}^{\sigma_{m S E}^{2}}=\frac{2 M \sigma_{A S E}^{2}}{P|A(0, \mathrm{t})|^{2}} \\
& \underset{\substack{\theta_{\text {AZ }-m \text { QAM }}^{2} \\
\sigma_{\text {Q }}^{2}}}{2}=\frac{16 M \sigma_{A S E}^{2}}{3 P|A(0, t)|^{2}}
\end{aligned}
$$

It is obvious from Eq.(10); RZ-mQAM signal is more affected by ASE noises at lower powers than mQAM signal. This phenomenon is due to mQAM wave has power content higher than RZ-mQAM wave.

In fiber-optics telecommunication systems, PCTWs signals are synchronously mixed to recover a received wave with high SNR and low NPN. The field of received mQAM signal can be expressed by: 


$$
\begin{aligned}
E_{r}(M L, t) & =E(M L, t)+\left[E^{*}(M L, t)\right]^{*} \\
= & E(0, t)\left[\exp \left(j \theta_{\text {SPM }}^{+ \text {ASE }}\right)+\exp \left(-j \theta_{\text {SPM }}^{*+A S E}\right)\right]
\end{aligned}
$$

The random NPN that rests after synchronous combining is:

$$
\delta \theta_{\mathrm{r}}=\frac{\theta_{\mathrm{SPM}}^{+\mathrm{ASE}}-\theta_{\mathrm{SPM}}^{*+\mathrm{ASE}}}{2}
$$

If the optical links are similar and launched powers are same, NPN variances of PCTWs are approximately equal. Variances of NPN for both RZ-mQAM and mQAM are reduced to:

$$
\begin{aligned}
& \sigma_{\mathrm{r}_{-} \mathrm{RZ}-\mathrm{mQAM}}^{2} \cong \frac{\sigma_{\theta_{\mathrm{SPM} \_\mathrm{RZ}-\mathrm{m} Q \mathrm{AMM}}^{\mathrm{ASE}}}^{2}+\sigma_{\theta_{\mathrm{RZ}-\mathrm{mQAM}}^{\text {ASE }}}^{2}}{2} \\
& =\frac{3 \mathrm{M}(\mathrm{M}+1)}{16} \gamma^{2} \mathrm{~L}_{\text {eff }}{ }^{2} \mathrm{P}_{\mathrm{o}} \sigma_{\mathrm{ASE}}^{2}|\mathrm{~A}(0, \mathrm{t})|^{2}+\frac{8 \mathrm{M} \sigma_{\mathrm{ASE}}^{2}}{3 \mathrm{P}_{\mathrm{o}}|\mathrm{A}(0, \mathrm{t})|^{2}},
\end{aligned}
$$

and

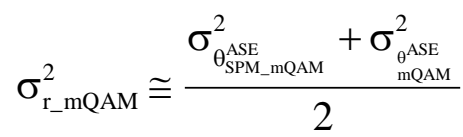

$$
\begin{aligned}
& =\frac{\mathrm{M}(\mathrm{M}+1)}{2} \gamma^{2} \mathrm{~L}_{\mathrm{eff}}^{2} \mathrm{P}_{\mathrm{o}} \sigma_{\mathrm{ASE}}^{2}|\mathrm{~A}(0, \mathrm{t})|^{2}+\frac{\mathrm{M} \sigma_{\mathrm{ASE}}^{2}}{\mathrm{P}_{\mathrm{o}}|\mathrm{A}(0, \mathrm{t})|^{2}} ;
\end{aligned}
$$

\section{SETUP OF PROPOSED SYSTEM}

Figure 2 depicts the setup of the SM RZ-coded PCTWs system. The setup involves two of RZmQAM modulators, similar optical links, and coherent receivers. In the transmitter site, a laser diode is used to supply the optical carrier of PCTWs. In the setup, the light beam with the wavelength of 1553.5 $\mathrm{nm}$ and linewidth of $105 \mathrm{HZ}$ is split by $3 \mathrm{~dB}$ coupler and directly provided to 4QAM modulators that utilize two Mach-Zehnder modulators (MZMs) for producing 4QAM waves. One pseudo-random binary sequence (PRBS) generator gives data, which is coded by mQAM mapper to form mQAM symbol and its phase-conjugated replica. For generating optical mQAM signal, in-phase(I) component run upper MZM while the quadrature-phase $(\mathrm{Q})$ component applied to lower MZM. In a similar way, another optical modulator generates a phase-conjugated wave. However, the phase of Q-component is shifted by 180 o before applying to lower MZM. RZ-mQAM signal is created by putting one MZM after the mQAM modulator. Sinusoidal wave generator, which oscillates at the symbol rate, derive this MZM. Sequentially, twin signals are delivered to optical links. Each span includes SMF, two DCFs, and EDFA. The SMF and DCF parameters are listed in Table I. Here the parameters of SMF, such as dispersion, and attenuation coefficients, are the same as the parametrs of standard SMF (SMF 28) [3031]. Accordingly, the accumulated dispersion over SMF is $960 \mathrm{ps} / \mathrm{nm}(16 \mathrm{ps} / \mathrm{nm} . \mathrm{km} \times 60 \mathrm{~km})$. The symmetrical dispersion plan is implemented by two DCFs. THE first DCF is located at the start of the span for compensating half dispersion with accumulated dispersion of $-480 \mathrm{ps} / \mathrm{nm}(-160 \mathrm{ps} / \mathrm{nm} . \mathrm{km} \times$ $3 \mathrm{~km}$ ) and another DCF is placed after SMF for compensating rest of dispersion. the EDFA is designed to compensate for the losses and it is placed at the end of the span 


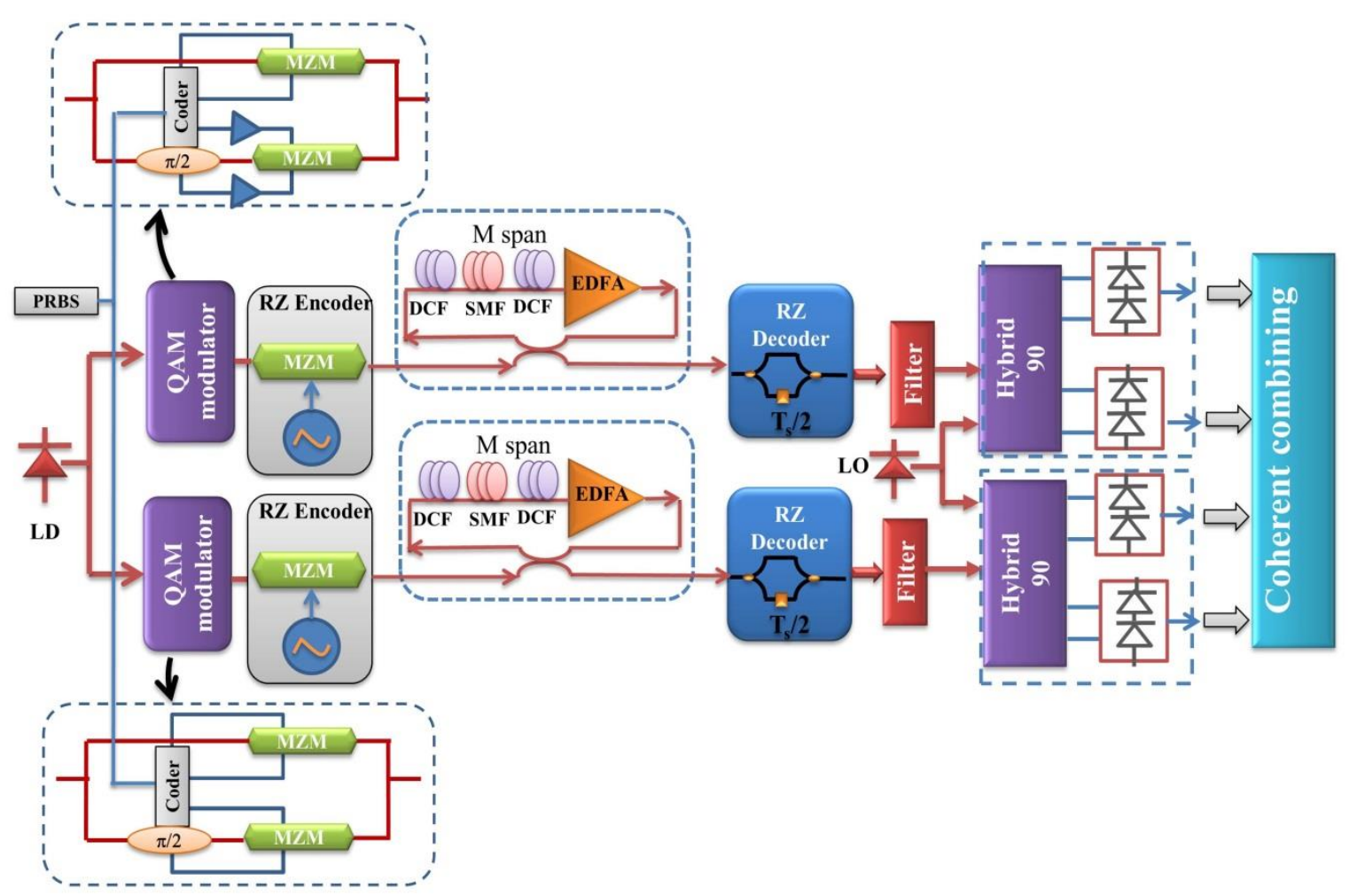

FIG. 2. DIAGRAM OF SM RZ-CODED PCTWS SYSTEM.

TABLE 1. PARAMETERS OF SMF and DCF

\begin{tabular}{ccc}
\hline Parameters & SMF & DCF \\
\hline Attenuation coefficient & $0.2 \mathrm{~dB} / \mathrm{Km}$ & $0.5 \mathrm{~dB} / \mathrm{Km}$ \\
\hline Dispersion coefficient & $16 \mathrm{ps} /(\mathrm{nm} \cdot \mathrm{km})$ & $-160 \mathrm{ps} /(\mathrm{nm} \cdot \mathrm{km})$ \\
\hline Nonlinearity coefficient & $1.3 \mathrm{~W}^{-1} \mathrm{~km}^{-1}$ & $5.2 \mathrm{~W}^{-1} \mathrm{~km}^{-1}$ \\
\hline Length & $60 \mathrm{~km}$ & $3 \mathrm{~km}$ \\
\hline
\end{tabular}

At the receiver side, received signals are initially applied to Mach-Zehnder interferometers (MZIs) for restoring mQAM waves (see Fig. 2). The time delay of MZIs is adjusted to $\mathrm{Ts} / 2$ in order to interfere symbols with their delayed duplicate. mQAM signal is produced at the constructive port. After that, the wave is refined and mixed with a local oscillator(LO) inside a coherent receiver to recover the transported waves. Then, received signals are mixed to eliminate SPM noise and to improve the quality of the received signal.

\section{RESULTS AND DISCUSSION}

The impact of the proposed scheme on enhancing the performance of PCTWs in the SDM transmission system is examined. The NPN is analytically evaluated regarding the analytical model in Section 2. Furthermore, performances of both SM PCTWs RZ-4QAM and SM PCTWs 4QAM systems are numerically carried out. In all systems, twin signals are modulated with the rate of 20Gsymbol/s.

To discover the effect of RZ-coded twin waves on SPM noise, Fig 3 demonstrates NPN variances of proposed and 4QAM schemes for a power range of -11 to $9 \mathrm{dBm}$. The results are calculated by the analytical model at a link length of $1800 \mathrm{~km}$ (30spans). Figure 3 shows that, at first, the NPN variances of both systems are decreased with raising channel power till the optimum level then the NPN increases with the higher power. This is because the RZ-4QAM twin waves have lower average power than for 4QAM signals. Furthermore, it can be observed from the figure, at power levels lower than $-3 \mathrm{dBm}$, the 
variance of phase noise of the proposed method is more than that for the PCTWs 4QAM system. Whatever, with increasing the channel power, phase noise variance is considerably dropped and it reaches value lower than that for the 4QAM scheme. lowest values of NPN variances are passed $1 \mathrm{dBm}$ for the proposed scheme and $-1 \mathrm{dBm}$ for the 4QAM system. This signifies that the proposed system can send optical waves with less phase noise at higher channel power levels.

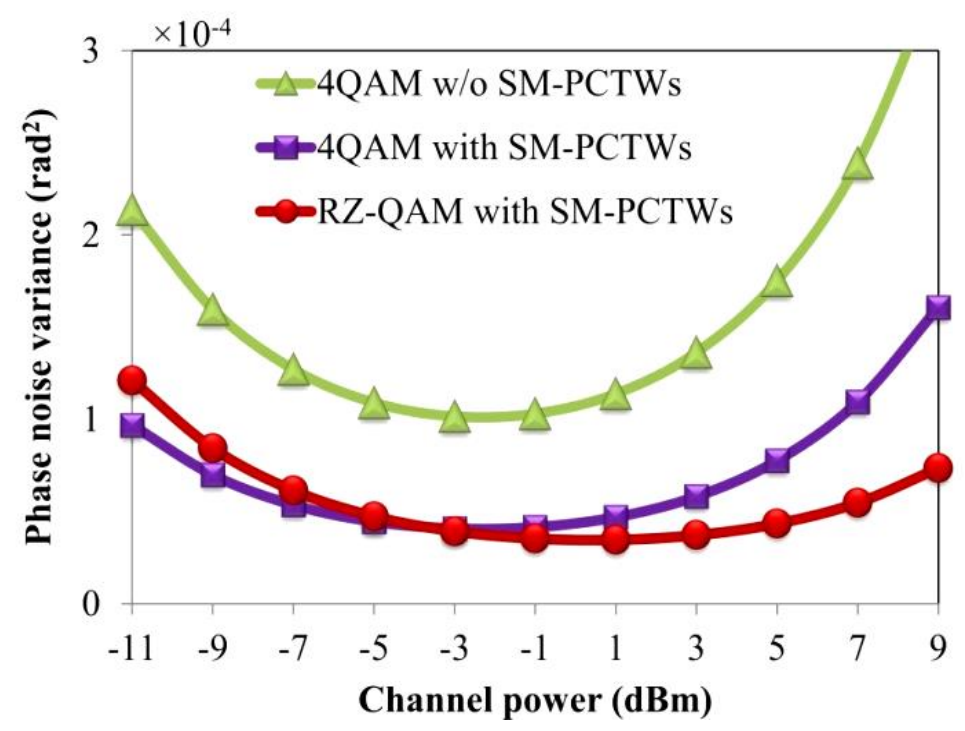

FIG. 3. INFLUENCE OF USING RZ-CODING ON NONLINEAR PHASE NOISE IN SM-PCTWS SYSTEMS

The performance enhancement of proposed systems when the RZ-4QAM format is employed as compared with the 4QAM system is illustrated in Fig. 4. The SNR is plotted versus channel power at a link length of $1800 \mathrm{~km}$. Generally, SNRs for both RZ-4QAM and 4QAM systems without and with PCTWs technique are firstly elevated when power level raises. Notwithstanding, as channel power exceed optimum power level for the systems, the SNR lowers because the SPM and its interaction with ASE noise are more influence at higher channel power. Besides, Figure 4 displays that using the PCTWs method noticeably increases SNR for 4QAM and RZ-4QAM schemes. It can be noted that for power range less than $-3 \mathrm{dBm}$, SNR of the proposed system is smaller, while it is greater for the range larger than $-3 \mathrm{dBm}$ as compared to the 4QAM system. For our proposed system, the maximum SNR is $25 \mathrm{~dB}$ while it is $23 \mathrm{~dB}$ for the conventional scheme with the PCTWs method. Thus, in contrast to the 4QAM system, the SNR of PCTWs RZ-4QAM scheme is higher by about $6.5 \mathrm{~dB}$. This phenomenon due to the RZ-mQAM wave has average power and interference timeless than mQAM wave, leading to moderate induced phase noise and boost the SNR of received signal.

To explore the advantages of employing RZ coding on the 4QAM PCTWs system, Fig. 5; shows the bit error rate (BER) as opposed to the transmission reach for the RZ-4QAM and 4QAM schemes without and with the PCTWs method. Launched power is fixed at $1 \mathrm{dBm}$ and $-1 \mathrm{dBm}$ for RZ-4QAM and 4QAM schemes; respectively.

To varying the propagation distance, the number of the span is changed. As expected, the reshaping envelope of 4QAM PCTWs enables our proposed scheme to transmit data for longer distances in comparison to the 4QAM system. Although the pre-forward-errorcorrection (pre-FEC) threshold of BER is $3.8 \times 10^{-3}$ [32], the results are obtained at BER of $10^{-5}$ to show the effect of the proposed method on transmission performance. The 
transmission reach is lengthened from $4500 \mathrm{~km}$ for the 4 QAM system $9000 \mathrm{~km}$ for the proposed scheme. In addition, transmission reach scores $8000 \mathrm{~km}$ for the SM PCTWs 4QAM system. Accordingly, the propagation reach is extended by $77.8 \%$ and $100 \%$ for SM PCTWs 4QAM and RZ-4QAM systems.

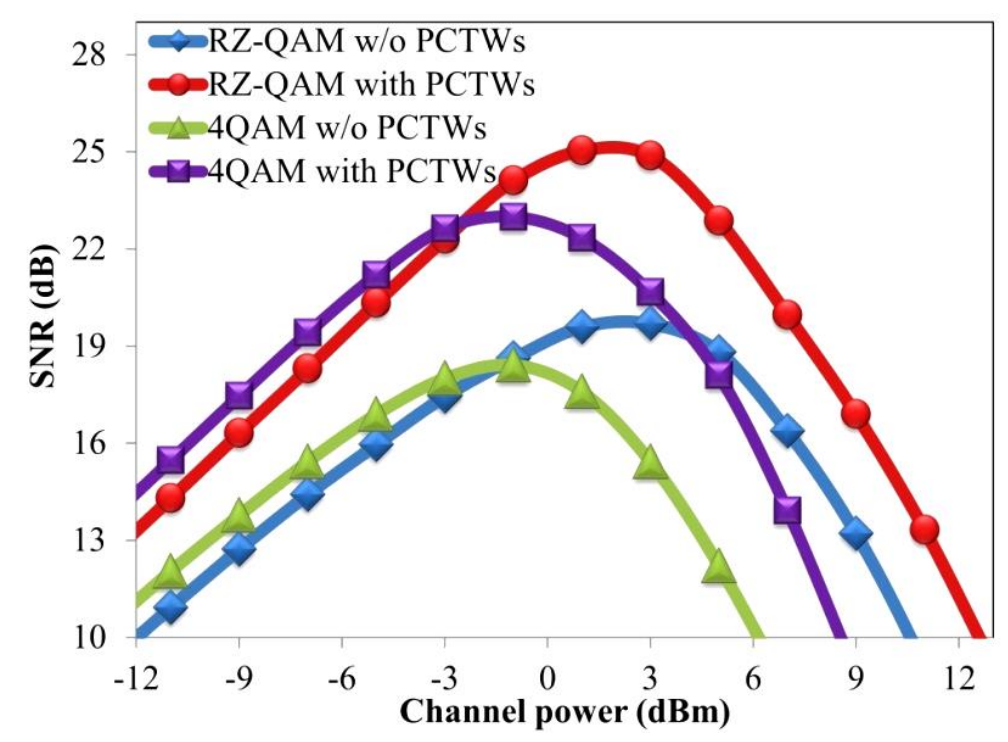

\section{FIG. 4. EFFECT OF PROPOSED TECHNIQUE ON PERFORMANCE OF SPATIAL MULTIPLEXED TWIN WAVES SCHEME}

In order to study receiver sensitivity of the proposed system, BER as opposed to optica1 signa1-to-noise ratio (OSNR) is displayed in Fig. 6. The results are achieved at the link extent of $1800 \mathrm{~km}$ and optimum power for all systems. As estimated, the results exhibit that receiver sensitivity is noticeably enhanced once the RZ-coded PCTWs technique is used in the 4QAM system. The required OSNRs at $B E R=10^{-5}$ are $11.2 \mathrm{~dB}$ for the 4QAM scheme and $4.3 \mathrm{~dB}$ for the proposed scheme. From the results, it can be concluded that the required OSNR is highly improved where it is enhanced by $6.9 \mathrm{~dB}$ over the traditional system.

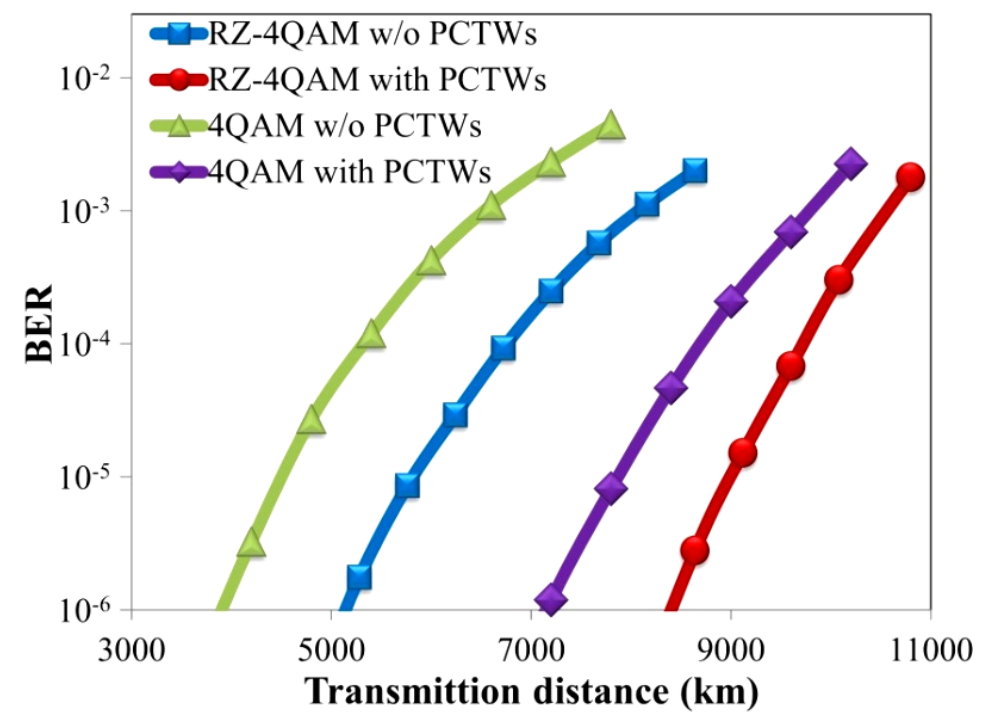

FIG. 5. BER AGAINST TRANSMISSION LENGTH FOR SM-PCTWS SCHEMES. 


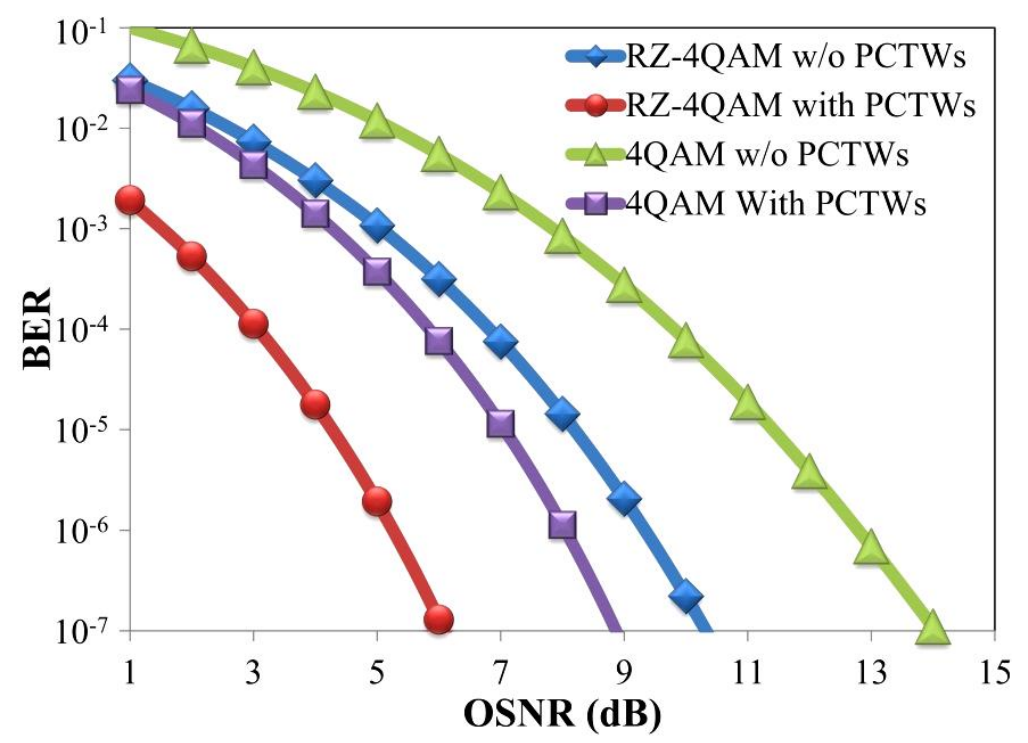

FIG. 6: BER VERSUS OSNR IN SM-PCTWS SYSTEMS

\section{CONCLUSIONS}

A novel and efficient method to improve the performance of the SDM system has been proposed and examined. Improving PCTWs performance has been accomplished by forming an envelope of PCTWs using RZ-coder. In the proposed approach, the twin waves have been modulated by the same laser source and then launched into similar fiber links. The received signals have been coherently mixed to remove the nonlinear effects. The impact of using the RZ code on the efficacy of the PCTWs method has been analytically modelled and displayed. The results reveal that coding the envelope of twin waves substantially boosts system performance that employs the PCTWs method. In comparison to the 4QAM scheme, SNR of the proposed scheme is raised by $6.5 \mathrm{~dB}$.

Additionally, at BER of $10^{-5}$, the achievable reach has been lengthened by $100 \%$ while the transmission reach for the 4QAM scheme with PCTWs is extended by $77.8 \%$ over that for the 4QAM system. Thus, our proposed system can transfer the same information for longer distances.

\section{REFERENCES}

[1] D. Hillerkuss, R. Schmogrow, T. Schellinger, M. Jordan, M. Winter and G. Huber,"26 Tbit $\mathrm{s}^{-1}$ line-rate super-channel transmission utilizing all-optical fast Fourier transform processing," Nature photonics, vol. 5, p. 364, 2011.

[2] J. Sakaguchi, B. J. Puttnam, W. Klaus, Y. Awaji, N. Wada and A. Kann., "305 Tb/s space division multiplexed transmission using homogeneous 19-core fiber," Journal of Lightwave Technology, vol. 31, pp. 554-562, 2013.

[3] M. Qiu, Q. Zhuge, M. Chagnon, Y. Gao, X. Xu and M. Morsy-Osman,"Digital subcarrier multiplexing for fiber nonlinearity mitigation in coherent optical communication systems," Optics Express, vol. 22, pp. 18770-18777, 2014.

[4] R. Kudo, T. Kobayashi, K. Ishihara, Y. Takatori, A. Sano and Y. Miyamoto, "Coherent optical single carrier transmission using overlap frequency domain equalization for long-haul optical systems," Journal of Lightwave Technology, vol. 27, pp. 3721-3728, 2009. 
[5] T. Pfau, S. Hoffmann, O. Adamczyk, R. Peveling, V. Herath and M. Porrmann,"Coherent optical communication: Towards realtime systems at $40 \mathrm{Gbit} / \mathrm{s}$ and beyond," Optics Express, vol. 16, pp. 866-872, 2008 .

[6] M. Nakazawa, K. Kikuchi and T. Miyazaki, High spectral density optical communication technologies vol. 6: Springer Science \& Business Media, 2010.

[7] Z. Zheng, X. Lv, F. Zhang, D. Wang, E. Sun and Y. Zhu, "Fiber nonlinearity mitigation in 32-Gbaud 16QAM Nyquist-WDM systems," Journal of Lightwave Technology, vol. 34, pp. 2182-2187, 2016.

[8] J. K. Hmood, S. W. Harun, S. D. Emami, A. Khodaei, K. A. Noordin and H. Ahmad,"Performance analysis of an all-optical OFDM system in presence of non-linear phase noise," Optics express, vol. 23, pp. 3886-3900, 2015.

[9] E. Giacoumidis, I. Aldaya, M. A. Jarajreh, A. Tsokanos, S. T. Le and F. Farjady,"Volterra-based reconfigurable nonlinear equalizer for coherent OFDM," IEEE Photonics Technology Letters, vol. 26, pp. 1383-1386, 2014.

[10] X. Zhu and S. Kumar, "Nonlinear phase noise in coherent optical OFDM transmission systems," Optics express, vol. 18, pp. 7347-7360, 2010.

[11] X. Liu, A. Chraplyvy, P. Winzer, R. Tkach, and S. Chandrasekhar, "Phase-conjugated twin waves for communication beyond the Kerr nonlinearity limit," Nature Photonics, vol. 7, p. 560, 2013.

[12] L. B. Du, M. M. Morshed, and A. J. Lowery, "Fiber nonlinearity compensation for OFDM super-channels using optical phase conjugation," Optics express, vol. 20, pp. 19921-19927, 2012.

[13] T. Yoshida, T. Sugihara, K. Ishida, and T. Mizuochi, "Spectrally-efficient dual phase-conjugate twin waves with orthogonally multiplexed quadrature pulse-shaped signals," in Optical Fiber Communication Conference, p. M3C 6, 2014.

[14] M. Morshed, L. B. Du, and A. J. Lowery, "Mid-span spectral inversion for coherent optical OFDM systems: Fundamental limits to performance," Journal of Lightwave Technology, vol. 31, pp. 58-66, 2013.

[15] I. Phillips, M. Tan, M. Stephens, M. E. McCarthy, E. Giacoumidis, S. Sygletos, et al., "Exceeding the nonlinear-Shannon limit using Raman laser based amplification and optical phase conjugation," in OFC 2014, 2014, pp. 1-3, 2014.

[16] K. Cheng and J. Conradi, "Reduction of pulse-to-pulse interaction using alternative RZ formats in 40-Gb/s systems," IEEE Photonics Technology Letters, vol. 14, pp. 98-100, 2002.

[17] R. Tripathi, R. Gangwar, and N. Singh, "Reduction of crosstalk in wavelength division multiplexed fiber optic communication systems," Progress In Electromagnetics Research, vol. 77, pp. 367-378, 2007.

[18] S. E. Mirnia, A. Zarei, S. D. Emami, S. W. Harun, H. Arof and H. Ahmad., "Proposal and performance evaluation of an efficient RZ-DQPSK modulation scheme in all-optical OFDM transmission systems," Journal of Optical Communications and Networking, vol. 5, pp. 932-944, 2013.

[19] J. K. Hmood, K. A. Noordin, S. W. Harun, and H. M. Shalaby, "Mitigation of phase noise in all-optical OFDM systems based on minimizing interaction time between subcarriers," Optics Communications, vol. 355, pp. 313-320, 2015.

[20] J. Hmood, K. Noordin, H. Ahmad, and S. Harun, "Performance improvement of all-optical OFDM systems based on combining RZ coding with m-array QAM," JOURNAL OF OPTOELECTRONICS AND ADVANCED MATERIALS, vol. 17, pp. 33-38, 2015.

[21] D. Van Den Borne, S. Jansen, S. Calabro, N. Hecker-Denschlag, G. Khoe, and H. de Waardt, "Reduction of nonlinear penalties through polarization interleaving in $2 / \mathrm{spl}$ times/10 $\mathrm{gb} / \mathrm{s}$ polarization-multiplexed transmission," IEEE photonics technology letters, vol. 17, pp. 1337-1339, 2005.

[22] X. Liu, S. Chandrasekhar, P. Winzer, R. Tkach, and A. Chraplyvy, "Fiber-nonlinearity-tolerant superchannel transmission via nonlinear noise squeezing and generalized phase-conjugated twin waves," Journal of Lightwave Technology, vol. 32, pp. 766-775, 2014.

[23] S. T. Le, M. E. McCarthy, N. Mac Suibhne, A. D. Ellis, and S. K. Turitsyn, "Phase-conjugated pilots for fibre nonlinearity compensation in CO-OFDM transmission," Journal of Lightwave Technology, vol. 33, pp. 1308-1314, 2015.

[24] X. Liu, H. Hu, S. Chandrasekhar, R. Jopson, A. Gnauck and M. Dinu., "Generation of 1.024-Tb/s NyquistWDM phase-conjugated twin vector waves by a polarization-insensitive optical parametric amplifier for fiber-nonlinearity-tolerant transmission," Optics Express, vol. 22, pp. 6478-6485, 2014. 
[25] S. Le, M. McCarthy, S. Turitsyn, I. Phillips, D. Lavery and T. Xu,"Optical and digital phase conjugation techniques for fiber nonlinearity compensation," in 2015 Opto-Electronics and Communications Conference (OECC), pp. 1-3, 2015.

[26] J. K. Hmood and S. S. Radhi, "Influence of physical dimensions on efficiency of phase-conjugated twin waves technique in coherent optical communication systems," Optics Communications, vol. 428, pp. 113119, 2018.

[27] J. S. Tavares, L. M. Pessoa, and H. M. Salgado, "Nonlinear compensation assessment in few-mode fibers via phase-conjugated twin waves," Journal of Lightwave Technology, vol. 35, pp. 4072-4078, 2017.

[28] J. K. Hmood, K. A. Noordin, and S. W. Harun, "Effectiveness of phase-conjugated twin waves on fiber nonlinearity in spatially multiplexed all-optical OFDM system," Optical Fiber Technology, vol. 30, pp. 147-152, 2016.

[29] A. Ellis, S. Le, M. McCarthy, and S. Turitsyn, "The impact of parametric noise amplification on long haul transmission throughput," in 2015 17th International Conference on Transparent Optical Networks (ICTON), pp. 1-4, 2015.

[30] Kuchta and Daniel M,"Error-free $56 \mathrm{~Gb} / \mathrm{s}$ NRZ modulation of a 1530-nm VCSEL link." Journal of Lightwave Technology 34.14, pp. 3275-3282, 2016.

[31] Gao and Junming,"40-Gb/s star 16-QAM transmitter based on single dual-drive Mach-Zehnder modulator." Chinese Optics Letters 7.2, pp. 109-111, 2016.

[32] Wang and Yuanquan, "Demonstration of 575-Mb/s downlink and 225-Mb/s uplink bi-directional SCM-WDM visible light communication using RGB LED and phosphor-based LED." Optics express 21.1, pp. 1203-1208, 2013. 